\title{
Proposed protocol to keep COVID-19 out of hospitals
}

\author{
n Cite as: CMAJ 2020 March 9;192:E264-5. doi: 10.1503/cmaj.1095852
}

Posted on cmajnews.com on February 20, 2020

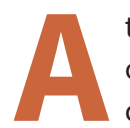

t-home testing and monitoring of possible COVID-19 cases could ease pressure on hospitals and emergency services and prevent the spread of infection, say experts. Public health officials, hospital leaders and paramedics in the Champlain Local Health Integration Network in Ontario are working on a protocol to do just that.

Under the proposed protocol, people suspected to have COVID-19 would be assessed, swabbed and monitored at home by specially trained paramedics in protective equipment, rather than being tested in hospital. According to Dr. Robert Cushman, acting medical officer of health for Renfrew County and District Health Unit, the paramedics could also monitor mild to moderate cases of COVID-19 over time, allowing patients who don't require hospitalization to recuperate at home. Approval of the protocol is still pending.

Currently, most people with suspected COVID-19 are told to go to the emergency department by ambulance or personal vehicle, and to notify health providers that they're coming. But according to Cushman, this approach can be inconvenient and "raises the possibility of further infection." He notes that Renfrew County hasn't had any cases that meet the criteria for testing yet, "so this is forward thinking."

Mike Nolan, chief paramedic and director of emergency services for Renfrew County, says home assessment for COVID-19 would "save precious resources." Paramedics wouldn't need to transport patients to hospital unnecessarily and then need to do "all the decontamination and disinfection of the ambulance that goes along with that," he explains.

England's National Health Service began piloting a similar home-testing program for

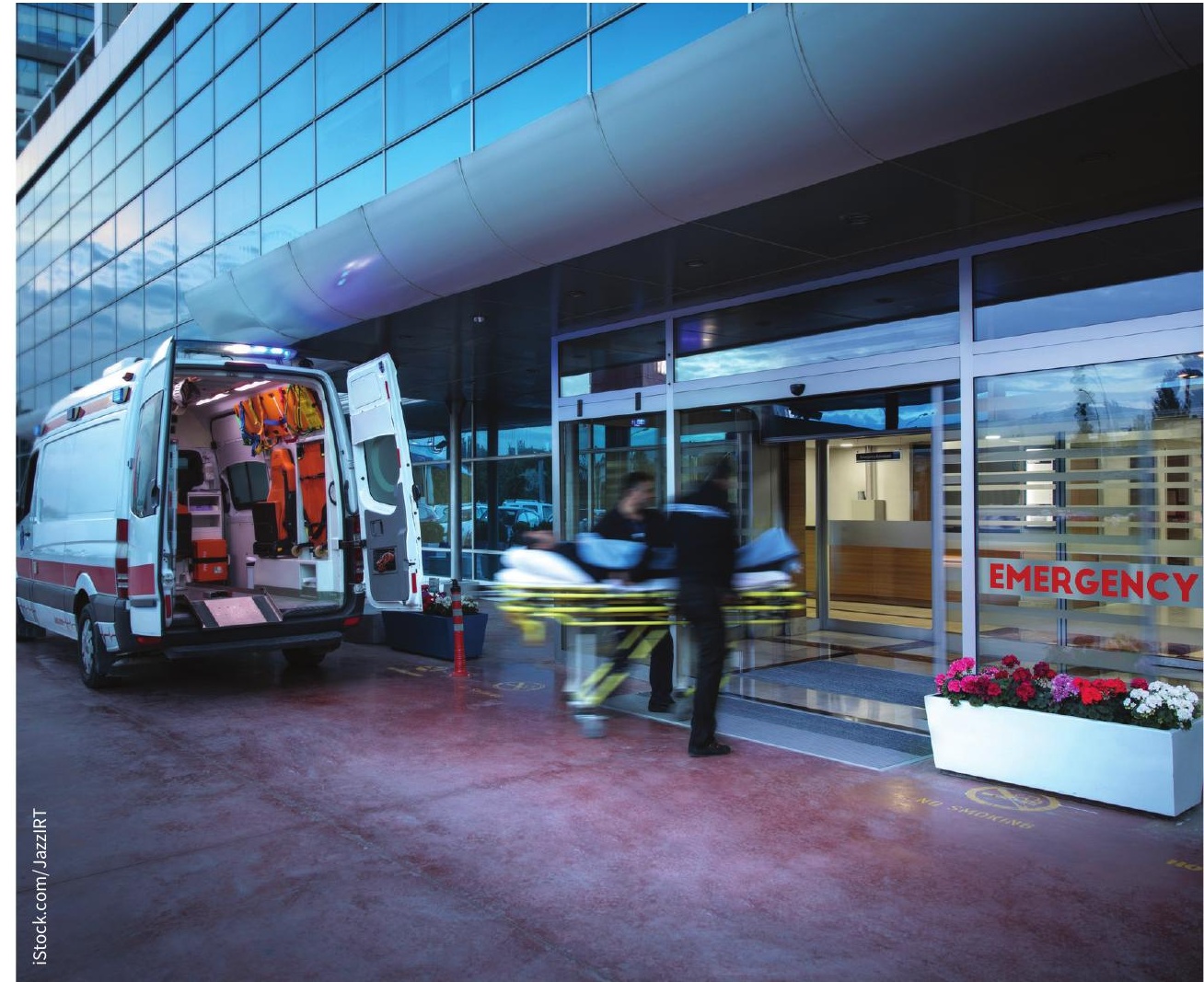

Sending people to hospital for COVID-19 testing "raises the possibility of further infection," says Dr. Robert Cushman of Renfrew County and District Health Unit.

COVID-19 in London last month. The pilot launched after 25 of the city's ambulances were decommissioned for decontamination in a single afternoon after transporting suspected cases. As of mid-February, the program has tested more than 130 patients.

At-home testing is a "very good idea," says Dr. David Fisman, a professor of epidemiology at the Dalla Lana School of Public Health in Toronto. According to Fisman, coronaviruses like severe acute respiratory syndrome (SARS) and COVID-19 become a problem "partly because health care institutions seem to facilitate their transmission."
Many Canadian emergency departments are already running at maximum capacity. And during the 2003 SARS outbreak, the virus spread quickly within health facilities.

According to Fisman, "the idea of saving clinical facilities for care provision, and screening people for infection in ways that don't require them coming in and clustering together with a bunch of uninfected patients seems commonsensical."

Close to 40 people have visited the emergency department at Sunnybrook Health Sciences Centre in Toronto with concerns about COVID-19, says Dr. Jerome 
Leis, a general internal medicine and infectious disease physician at the hospital. Twenty-one of those people warranted testing, and one person tested positive. Besides the person who tested positive, only one person had symptoms requiring hospitalization.

Leis says that his colleagues at other hospitals have seen even more patients asking for COVID-19 testing. He worries that the number will rise and overwhelm emergency department resources.

"Even mildly symptomatic patients can transmit this infection, so keeping them in home isolation is preferable," Leis says. "Even with our infection control protocols, there are usually a large number of individuals involved in the care of patients and more opportunities for exposure to occur [at the hospitals compared to at home]."

Some Canadian public health units already provide at-home testing for COVID19 , but this varies according to jurisdiction. Dr. Michael Finkelstein, acting director of communicable disease control at Toronto Public Health, says the unit conducts home testing only for close contacts of confirmed cases (as well as for confirmed cases to determine when they no longer need to be isolated). They advise everyone else, including people who recently travelled in China and have symptoms of respiratory illness, to go to an "appropriate setting that supports the safe use of N95 respirators during specimen collection." Most often, that means going to a hospital emergency department.

In Calgary, public health authorities will generally provide COVID-19 testing at a physician's office or at home for patients with mild symptoms, according to Tom McMillan, assistant director of communications for Alberta Health Services. He says patients and providers should call the province's 811 telehealth line to arrange testing.

While public health staff can provide home testing in cities like Calgary, Cushman says paramedics are better positioned to do so in eastern Ontario because "they are much more decentralized than we are in public health." In Renfrew County, for example, there are two public health offices and seven paramedic bases.

Paramedics in the Champlain Local Health Integration Network have experience assessing and monitoring patients in their homes, with a seasonal surge pilot program providing mobile care for 1541 patients suspected of having influenza in 2018-2019. The paramedics visited some patients daily, treated dehydration and performed on-site rapid tests for influenza. An evaluation of the pilot found that 99\% of patients avoided hospitalization. The program has since been adopted in Hamilton.

According to Nolan, the proposed COVID-19 protocol goes beyond the influenza surge program, involving technologies that allow paramedics to monitor patients remotely. Patients would be equipped with a device that would measure their temperature, blood pressure, heart rate, and oxygen saturation and feed those results to paramedics. That way, paramedics wouldn't have to put on protective equipment to check those signs daily, as they did with the flu program, says Nolan. Patients would be provided with a tablet to communicate securely with a health provider or public health official.

The program would also include an assessment to make sure that patients have support at home and closer monitoring for older patients and others at higher risk. Cushman notes that most people with COVID-19 don't require hospitalization. But since some cases can be fatal, "there's a fine line and there's a lot of clinical judgment that goes into monitoring their progress," he says.

Wendy Glauser, Toronto, Ont. 